\title{
Fuzzy adaptive sliding mode control of servo system based on the
}

\section{essential oil filling}

\author{
Wen YOU ${ }^{1, a}$, Xu-long ZHANG ${ }^{1, b^{*}}$, Hong-bo WEI ${ }^{1, c}$ \\ ${ }^{1}$ Department of Control Engineering, Changchun University of Technology, Changchun 130012, \\ China \\ ayouwen@ccut.edu.cn, ${ }^{\mathrm{b}}$ longyanzhuan@163.com, ${ }^{\mathrm{c}} 326984121 @ q q . c o m$
}

\section{Keywords:Essential oil filling,Sliding ModeControl,Fuzzy adaptive,High frequency tracking}

Abstract.To solve the problem that the tracking control precision is low when the essential oil filling linear servo system driven by Permanent magnet synchronous linear motor is under high frequency input signal, the fuzzy adaptive sliding mode control algorithm is designed. The sliding mode control is adopted to track, the fuzzy adaptive control is used for fuzzy approximation to the discontinuous control variable of traditional sliding mode controller as well as the compensation for the uncertainty of the actual operating system, the corresponding adaptive algorithm is also designed to make up for the deficiency of fuzzy controller, then effectively reducing the chattering. Simulation results show that the control algorithm can effectively improve the high frequency tracking characteristicof the servo system, accelerate the response speed and enhance the robustness.

\section{Introduction}

In the oil filling process for filling machine ,the claim must have a high feed speed,and can constantly adjust the feeding speed according to the different filling bottles in the shortest possible time.so as to ensure that the filling of high precision, high speed.The traditional filling method is difficult to meet the requirements, and the precision of filling technology usually need installation a fast response ability of micro displacement servo system in the filling machine.

The current study of filling machine servo system mainly in two aspects: high frequency response and high precision[1].The realization of the high frequency response needs innovation and improvement to micro-feed actuators.But the high precision need through effective control algorithm to achieve.In terms of FTS drivers, piezoelectric stack, the foreign scholars have done a lot of research,like lorentz force drives,maxwell's force drive and its compound drive way.And domestic scholars also have done a lot of research on high frequency response and ultra high frequency response,including tsinghua university,jilin university and national defense science and technology university and other colleges and universities.Mainly includes the PID control and repetitive control[2] and sliding mode control[3],etc.

This article convenient experiment to obtain larger trip,for the purpose of selection using linear motor as the driving part of the servo system.Due to the stroke and frequency response is contradictory. This leads to at the expense of high frequency response partly.In this paper,the various factors influencing the system bandwidth and frequency response are analyzed.And considering the linear motor drive characteristic and external disturbance and end disturbance and other factors,decided to apply the fuzzy adaptive sliding mode control algorithm to improve the precision of the servo system. 


\section{Linear motor mathematical model}

Ignore the loss of eddy current and magnetic hysteresis. When only considering fundamental component,d-p axis vector control model can be applied.And use the vector control mode that $i_{d}=0 A$.Get a d-p coordinates system model of permanent magnet linear synchronous motor by Park transformation. That is

$\left\{\begin{array}{l}\frac{d i_{q}}{d t}=\frac{R}{L_{q}} i_{q}-\frac{\pi \phi_{m}}{\tau L_{q}} v+\frac{1}{L_{q}} u_{q} \\ \frac{d v}{d t}=\frac{3 n_{p} \pi \phi_{m}}{2 m \tau} i_{q}-\frac{f_{d}}{m}\end{array}\right.$

In the equation, $R$ is primary resistance, $\phi_{m}$ is permanent magnet flux linkage, $\tau$ is primary notch, $i_{q}, u_{q}, L_{q}$ are the current, voltage and inductance of the $\mathrm{q}$ axis, $\mathrm{m}$ is equivalentmass, $n_{p}$ is pole pairs, $v$ is velocity, $f_{d}$ is the disturbance of the system.

\section{An adaptive fuzzy sliding mode control system}

The servo control system of fuzzy adaptive sliding mode structure diagram is shown in fig. $1 . \gamma$ is the reference position.Control problem is to find the appropriate control rules.Makes the position of the motor's activecell can track the given high frequency sinusoidal reference signal.

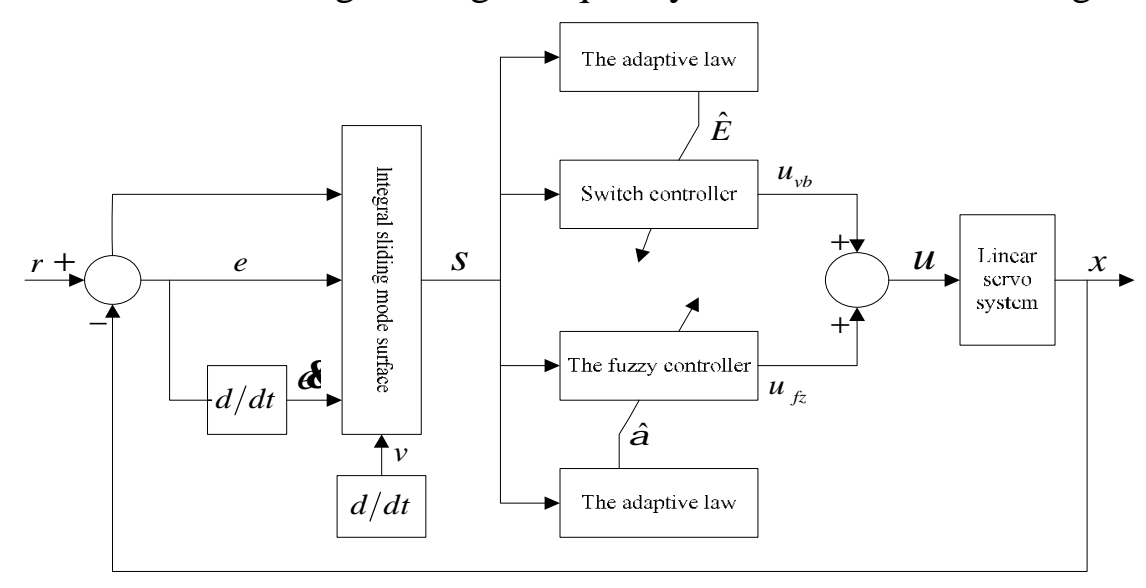

Fig.1 Adaptive fuzzy sliding mode linear servo control system

\section{The design of fuzzy sliding mode control}

Assuming that the system is the second order SISO nonlinear system.That is

$\ddot{x}=f(x, t)+g(x, t) u(t)+d(t)(2)$

In the equation, $f, g$ is nonlinear position function. And $g>0 . u(t)$ is control law. $d(t)$ is disturbance.Assuming that $x_{d}(t)$ isposition command[4], The tracking error of the nonlinear system is $e(t)=x(t)-x_{d}(t)$.Define the integral sliding mode surface is 


$$
S(t)=\dot{x}(t)-\int_{0}^{t}\left[\ddot{x_{d}}-k_{1} e(t)-\dot{k}_{2} e(t)\right] d t
$$

In the equation, $k_{1}, k_{2}$ is the normal number of non-zero.If the system state trajectory reached the sliding mode surface shows system meet the existence condition and reach the sliding mode.Then $S(t)=\dot{s}(t)=0$, that is

$$
\ddot{x}(t)-\ddot{x_{d}}(t)+k_{1} \dot{e}(t)+k_{2} e(t)=0
$$

If you select the appropriate gain,ensure that Eq.4 meet the Hurwitz conditions. The root is in the left half plane of the complex plane.That can make the tracking error to zero.In this paper,Based on the parameter setting method to get the right value of $k_{1}$ and $k_{2}$.According to the Eq.2,we can get

$$
u(t)=\frac{-f(x, t)-d(t)-\ddot{x_{d}}-k_{1} \dot{e}-k_{2} e}{g(x, t)}
$$

Dynamic characteristics and the external disturbance of servo system are unknown,so, $f, g$ and $d$ is uncertain.It makes $u(t)$ difficult to obtain. So we need to use fuzzy control approach $u(t)$.Using switching function $C(t)$ as the input of fuzzy controller. $u_{f_{z}}$ is the output.So the control rules of fuzzy controller is

$$
\begin{aligned}
& u_{N M}\left(x_{i}\right)=\exp \left\{-\left[\left(x_{i}+\pi / 6\right) /(\pi / 24)\right]^{2}\right\} \\
& u_{N S}\left(x_{i}\right)=\exp \left\{-\left[\left(x_{i}+\pi / 12\right) /(\pi / 24)\right]^{2}\right\} \\
& u_{Z}\left(x_{i}\right)=\exp \left\{-\left[x_{i} /(\pi / 24)\right]^{2}\right\} \\
& u_{P S}\left(x_{i}\right)=\exp \left\{-\left[\left(x_{i}-\pi / 12\right) /(\pi / 24)\right]^{2}\right\} \\
& u_{P M}\left(x_{i}\right)=\exp \left\{-\left[\left(x_{i}-\pi / 6\right) /(\pi / 24)\right]^{2}\right\}
\end{aligned}
$$

Take its membership function are the negative(NM), negative small (NS), zero (Z), is positive small (PS), the positive median (PM).

Using these five rules of membership function for fuzzy sliding mode.Using the gravity method to anti-fuzzification[5].Get the Output of fuzzy controller system is

$$
u_{f z}(S)=\frac{\sum_{i=1}^{m} \omega_{i} \alpha_{i}}{\sum_{i=1}^{m} \omega_{i}}(6)
$$

In the equation, $\omega_{i}$ is the ith rule weights, $\alpha_{i}$ is the fuzzy output element set.

\section{The design of the fuzzy adaptive sliding mode control}

In fact, due to the system dynamic characteristic and external load disturbance may be unknown,or there is interference, so the feedback linearization control is difficult to achieve.This 
article uses the fuzzy adaptive sliding mode control to analog control law $u(t) . \alpha_{i}$ is adjustable parameter.So the control output is

$u_{f z}(S, \alpha)=\alpha^{T} \zeta^{T}$

In the equation, $\alpha=\left[\alpha_{1}, \alpha_{2}, \cdots, \alpha_{m}\right]^{T} ; \zeta=\left[\zeta_{1}, \zeta_{2}, \cdots, \zeta_{m}\right], \zeta_{i}=\frac{\omega_{i}}{\sum_{i=1}^{\omega_{i}} \omega_{i}}$

According to the fuzzy approximation theory, the optimal fuzzy system $u_{f z}(S, \alpha)$ is exist.Then $u(t)=u_{f z}(S, \alpha)+\varepsilon=\alpha^{T} \zeta^{T}+\varepsilon$

There, $\varepsilon$ is approximate error.Then

$u_{f z}(S, \hat{\alpha})=\hat{\alpha}^{T} \zeta^{T}$

There, $\hat{\alpha}$ is the approximating value of $\alpha$.

In fig. 1,the fuzzy adaptive sliding mode control law is

$u(t)=u_{f z}+u_{v s}$

There, $u_{v s}$ is switching control law.To compensate the error between $u$ and $u_{f z}$.

The Lyapunov function is

$V_{1}(t)=\frac{1}{2} S^{2}(t)+\frac{g(x, t)}{2 \eta_{1}} \tilde{\alpha}^{T} \tilde{\alpha}$

There, $\eta_{1}$ is positive real number.Then

$\dot{V}_{1}(t)=S(t) \dot{S}(t)+\frac{g(x, t)}{\eta_{1}} \tilde{\alpha}^{T} \dot{\tilde{\alpha}}=g(x, t) \tilde{\alpha}^{T}\left(s(t) \zeta+\frac{1}{\eta_{1}} \dot{\tilde{\alpha}}\right)+S(t) g(x, t)\left(u_{v s}-\varepsilon\right)$

To ensure $V_{1}(t)=0$, should adopt adaptive law that is

$\dot{\tilde{\alpha}}=\hat{\alpha}=-\eta_{1} S(t) \zeta$

The switching control law should be

$u_{v s}=-E(t) \operatorname{sgn}(S(t))$

In the switch control, because it is difficult to select the appropriate switch gain $E(t)$, so we often determine use the experience.Used the $\hat{E}(t)$ instead of $E(t)$.Then

$$
u_{v s}=-\hat{E}(t) \operatorname{sgn}(S(t))
$$


There, $\hat{E}(t)$ is the estimated value of $E(t)$.The error of estimation is $\tilde{E}=\hat{E}(t)-E(t)$. By using Lyapunov function, The closed loop system is

$$
V(t)=V_{1}(t)+\frac{g(x, t)}{2 \eta_{2}} \tilde{E}(t)
$$

Then

$$
\dot{V}(t)=-\hat{E}(t)|S(t)| g(x, t)-\varepsilon S(t) g(x, t)+\frac{g(x, t)}{\eta_{2}}[\hat{E}(t)-E(t)] \hat{E}(t)
$$

To make $\dot{V} \leq 0$, define the switch gain estimates is

$$
\hat{E}(t)=\eta_{2}|S(t)|
$$

\section{The factors that influence the bandwidth of the system analysis}

In a servo system, bandwidth is refers to the servo system can response the biggest sine wave frequency.Bandwidth is one of the most important indicators of performance as the measurement and characterization of the control system.Bandwidth embodies the system response speed of the input signal and interference suppression[6], etc.For servo system,on the one hand bandwidth , restricted by saturation, such as drive motor power and torque is limited, excessive bandwidth cannot be realized in practice,on the other hand it may make high-frequency interference by excessive bandwidth, up less than the effect of noise suppression, lead to the stability of the system are affected.In order to prevent the control noise of signal and other high frequency interference, and has to limit bandwidth[7].

Bandwidth can be achieved by adjusting the servo driver or control system parameters to adjust.It is also under the control of the rigidity of transmission chain and transmission clearance and load inertia.in addition to this, there are many other factors, according to the actual situation to adjust.

Bandwidth is usually not the only indicator of servo system, it also has And accuracy, stability and so on.We need balance the indicators, and combined with the specific purpose to comprehensive consideration.Does not the greater the bandwidth, the better.

From what has been discussed above, the design of the system bandwidth need comprehensive consideration of various factors of the system. The bandwidth of gain is by measuring the high frequency tracking and for interference suppression.

\section{The simulation and the result analysis}

The MATLAB simulation is used to check the frequency response, high frequency tracking performance and anti-interference performanceof the servo system. The simulation curve are fig.2, fig.3, fig.4 and fig.5. 

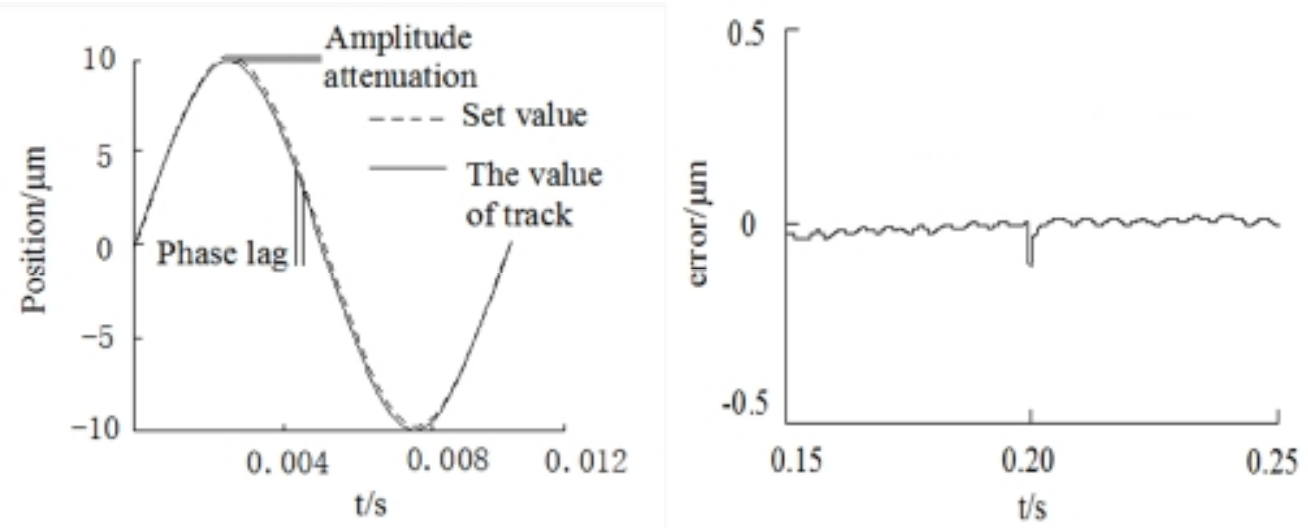

Fig.2 The position control curve in high frequencyFig.3 Position tracking error curve on Step disturbance
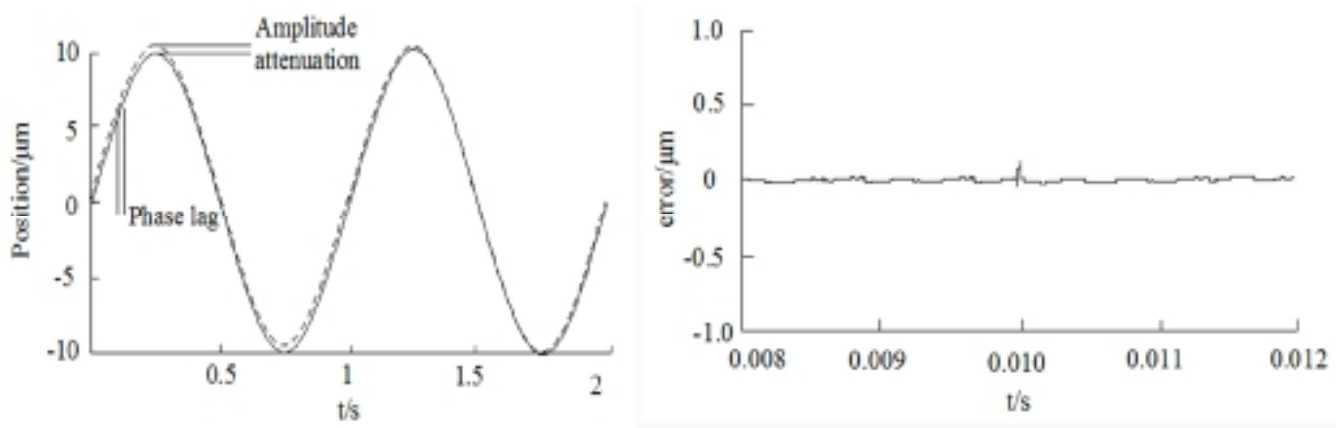

Fig.4 The position control curve inUltra high frequencyFig 5 Position tracking error curve

We can see from the fig.2, filling bottles of actual location is very close to the desired location.Fig. 3 isthe tracking error of curve which join the step disturbance at $0.2 \mathrm{~s}$. We can see from the error curve in the graph, fuzzy adaptive synovial control has better ability to resist disturbance.Stability of the tracking error within $0.2 \mathrm{um}$. This shows that the controller has higher tracking precision. We can see the tracking phase lag and amplitude attenuationclearly from the fig.4.Phase lag and amplitude attenuation are close to zero finally.Fig.5 is error curve which joins step disturbanceat $0.01 \mathrm{~s}$. We can see from fig. 5 , the error meet requirements.

\section{conclusions}

Tracking performance of the control system is directly affect the oil filling accuracy.Therefore, this article used in high frequency sinusoidal reference signal instead of the actual filling bottles of sports instruction, and designed a fuzzy adaptive sliding mode controller.Using Simulink to estimate the frequency response of the system. The simulation curve of the reference signal frequency which arethe high frequency and ultra high frequency proves the effectiveness of the controller. You can see from the simulation curve of the tracking error the controller has a better resistance to disturbance effect.In this paper, there are still two issues need further study,on the one hand, the uncertainty factors that influences the bandwidth of the system,on the other hand, we need to overcome the interference of filling process and other uncertain disturbances for improving the filling accuracy.

\section{References}

[1] Xiao-lan Huang. Rotary type filler control system based on PLC study [J]. Journal of wuhan university of science and technology, mechanical engineering. 2012

[2] $\mathrm{H}_{-} \infty$ tracking design for a class of decentralized nonlinear systems via fuzzy adaptive observer[J]. Journal of Systems Engineering and Electronics,2009,04:790-799. 
[3] Cong-bo Shi. When the nonlinear since the immunity control of accidental error of measurement of inhibition [J]. Journal of mechanical design and manufacturing. And dynamics of machinery, precision instruments, tsinghua university, Beijing, 2012 (7). 148-150.

[4] Fuzzy Adaptive Control of Stochastic Nonlinear Systems with Unknown Virtual Control Gain Function[J]. ACTA AUTOMATICA SINICA,2006,02:170-178.

[5] Li-xia An. Fuzzy sliding mode control based on t-s model of bionic research [J]. Journal of jiangsu university. 2014.

[6] Jing Jiang, En-hui Li. Fuzzy sliding mode control in the application of nc turntable servo system [J]. Journal of shenyang university of technology, 2014 (2): 61-65.

[7] Zeng-Ming Dong, ChunZhang, Bao-ku Su. High accuracy servo turntable control system the discussion of bandwidth design [J]. Journal of Chinese inertial technology. 2001 (2) : 55 to 59.

[8] Fuzzy Adaptive Control of Stochastic Nonlinear Systems with Unknown Virtual Control Gain Function[J]. ACTA AUTOMATICA SINICA,2006,02:170-178.

[9] LeiGu. Resonance problems in ac servo system research [J]. Journal of huazhong university of science and technology. 2012.

[10] Rong-Jong Wan,Chi-Min Lin,Chun-Fei Hu.Adaptive fuzzy sliding-mode control for electrical servo drive[J].Fuzzy Sets and Systems. 2003 (2) 